\title{
How Can loT Improve the Life-quality of Diabetes Patients?
}

\author{
Anne Marit Longva and Moutaz Haddara \\ Kristiania University College, Department of Technology, 32 Christian Krohgs Gate, 0186 Oslo, Norway
}

\begin{abstract}
The Internet of things (IoT) is revolutionizing several industries with unprecedented possibilities and opportunities. One of the industries that has a critical direct impact on humans, is the healthcare industry. While IoT technologies can be seen in medical supply chains within the healthcare sector, however, IoT technologies are still not widely adopted in other areas and services within the healthcare sector. The number of patients living with diabetes has increased drastically over the past four decades and is expected to increase even more over the next ones. The disease currently has no cure, and if the patients are not monitored and get the right treatment at the right time, it might end a patient's life. The IoT is said to be a game-changer for the healthcare industry and the aim of this paper is to review and investigate how IoT technologies and solutions can improve the quality of life and aid the people living with the chronic disease. Our findings suggest that IoT can aid in patient monitoring, including the continuous glucose monitoring, and also can assist in providing a healthier lifestyle for patients through activity and diet tracking. In addition, we have identified key challenges for IoT adoptions and implementations in healthcare within the scope of this research.
\end{abstract}

\section{Introduction}

It has been projected that within the next 10 years, the deaths related to chronic diseases will increase by $17 \%$, which compromises approximately sixty-four million people [1]. The symptoms of chronic diseases are highly variating, as well as their treatment and evolution. If the patients are not monitored and get the right treatment at the right time, it might end the patient's life abruptly. Among the common chronic diseases that can be managed with strict monitoring is diabetes [1]. The number of patients with diabetes has increased from 108 million in the 1980s to 422 million in 2014 [1]. Researchers reckon that the numbers will continue to rise over the next decades. Unfortunately, there is no cure for diabetes per today, but the disease can be treated with a strict diet, constantly measuring of blood glucose levels, physical activity, medication and regular screening and treatment [2].

The internet of things (IoT) is said to be a game changer for several industries [3], especially the healthcare industry [4], and many healthcare organizations are already investing in and adopting new digital technologies in order to create new value in the industry [5]. Researchers within this field are constantly looking for new and better ways to serve patients' needs in a reliable, secure and cost-effective way [6]. Integrating IoT in healthcare devices improves the quality and effectiveness of service, which will bring more value especially to the elderly and patients with chronic diseases [7]. IoT applied to care and monitoring of patients is increasingly common and is seeking to improve the quality of life for the patients [2]. The aim of this paper is to investigate how IoT-technologies can help in improving the life quality of people living with diabetes. We believe that many of the existing IoT solutions presented in this paper will in fact advance the life quality of diabetes patients by reducing the number of hospitals visits, providing a better overview of their general health and improving the communication between healthcare personnel and patients. The solutions also help the patients to keep track of their diet and activity levels, which is an important part of the treatment for diabetes patients. The implementation of IoT devices will also provide healthcare personnel the opportunity to access real-time data about their patients, making it easier to prescribe the right medication, intervention, and create the right treatment plan.

The remainder of the paper is organized as follows: our research methodology is presented in section 2. Section 3 demonstrates the main findings of this review. A discussion over the findings is provided in section 4, followed by conclusions and recommendations for future research in section 5 .

\section{Research methodology}

As this paper aims at reviewing the extant literature on IoT in healthcare with a focus on diabetes-related solutions, a systematic review [8] approach was conducted. Based on that, we first developed a search strategy and extracted the sources that are deemed relevant for this review. We searched through Google Scholar and Business Source Complete engines. A combination of keywords was used, including "Internet

* Corresponding author: moutaz.haddara@,kristiania.no 
of things", "IoT", "Healthcare" and "diabetes". In addition, some other keywords were also used, such as, "patient monitoring", "CGM", "RFID" and "Challenges".

The literature period was set between 2010 and June 2019 (at the time of writing this article). First, we were targeting only research from the leading information systems (IS) journals, what is known as "The basket of eight", namely: European journal of Information Systems, Information Systems Journal, Information Systems Research, Journal of the AIS, Journal of Information Technology, Journal of Strategic Information Systems and Management Information Systems Quarterly. However, we were not able to identify any relevant publications in the aforementioned journals. Thus, we decided to widen our search scope to include other IS journals and conferences, which also led to a very small number of papers. Later we widened our scope further to incorporate articles that have been published in engineering and medical journals and conferences, which are relevant to the focus of this study. Both authors went through all the papers in order to confirm their relevance to this research, and to classify the papers based on their main focus. Four main reoccurring areas were identified (see table 1), patient monitoring, continuous glucose monitoring, activity and diet tracking, and finally challenges of IoT adoptions within the diabetes treatment sector. In total, twentyeight papers were identified and reviewed, with some papers focusing on more than one topic, as shown in table 1.

\section{Findings}

\subsection{Internet of things}

Today, the Internet is an important part of our everyday life. It is expected that by 2020 , the number of connected devices to the Internet will cross the 50 billion mark [9]. The Internet of things (IoT) has the potential to add new dimensions to the traditional Internet through enabling communications with and among so-called smart objects, leading to a vision of "anytime, anywhere, anything" communications. A smart object is an autonomous, physical digital object supplemented with sensing, processing, storing and networking capabilities [4]. This makes them able to sense, store and interpret information that is stored locally, or within the other smart objects in the environment [10]. IoT will unquestionably have a high impact on several aspects of our everyday life and behaviour [11]. The main feature of IoT is the integration of several technologies and communications solutions. Identification and tracking technologies, enhanced communication protocols and distributed intelligence for smart objects, are just some of the other relevant features to the IoT [11]. The Internet of things has been defined as "the integration of all devices that connect to the network, which can be managed from the web and in turn, provide information in real-time to allow interaction with that use it" [1]. The idea behind the concept is the variety of things or objects around us, such as radio-frequency identification tags (RFID), mobile phones, sensors etc., that are able to interact and collaborate with each other and their neighbours through unique addressing systems [11].

\section{2 loT in healthcare}

Healthcare organizations all around the world are being transformed into more effective, coordinated and usercentred systems [9]. In fact, studies have projected that $87 \%$ of healthcare organizations will be using IoT technology in their facilities by the end of 2019 [12]. Healthcare professionals and executives around the world are acknowledging that the way healthcare is conducted and managed is now changing in drastic and fundamental ways [5]. Technological developments are helping healthcare to become more personalized, more cost-effective and scalable, and more capable of achieving successful outcomes than ever before. Previously, much research has been devoted to the exploration of technologies such as information technologies (IT) in supplementing and strengthening existing healthcare services. In particular, the Internet of Things has been extensively applied to connect existing medical resources and to provide smart, reliable and more effective healthcare service to the elderly and patients with a chronic illness [13]. The IoT-based healthcare systems connect all available resources as one network to perform different activities such as monitoring, diagnosing and even remote surgeries over the Internet [13]. The implementation of IoT in healthcare has led to more integrated interoperable healthcare services and easy access to medical records and related information for both patients and healthcare personnel [9]. The integration is also said to improve the quality and effectiveness of service, which brings especially high value for elderly and patients with chronic conditions and others that may require consistent supervision [7]. A smart healthcare device or system usually integrates sensing technologies with other devices and applications, which enables the healthcare system to monitor their patients [13]. For some patients, tracking data is vital. The use of IoT has been instrumental in delivering more valuable real-time data to doctors, which lessens the need for direct doctorpatient interaction [12]. One of the technologies that help in collecting real-time data is a wearable device. Patients are wearing these wearable devices in order to transmit data directly to physicians or other healthcare professionals, allowing them to view and monitor the vital signs and readings in real-time. The reading can be like heart rate, glucose levels, and may also provide them with fail detection algorithms [12]. This gives both medical staff and patients the possibility to monitor their levels consistently, providing both parties with more control and an enhanced feeling of safety. 


\subsubsection{Patient monitoring}

The rise of miniaturized sensors and actuators for monitoring, diagnostics, and therapeutic functions has opened up several new prospects to overcome healthcare challenges [14]. Networked sensors, worn on the body or embedded in the environment, make it possible to gather important information about a person's physical and mental health [15]. Sensor networks consist of a certain number of sensing nodes that are communicating in a multi-hop approach. Most of the suggested frameworks for remote health monitoring contain a three-layer architecture: A Wireless Body Area Network (WBAN) that is comprises various wearable sensors, which measure physiological parameters such as body temperature and blood pressure [15]. The sensors transmit the gathered information to a gateway server through a Bluetooth connection. Further on, the gateway turns this data into an observation and measurement file that is stored on a remote server (usually cloud-based) for later retrieval. Medical staff can access the stored data online through content service application [15]. Storage and access, medical data analysis and visualizations are all critical components of remote patient monitoring. In order to give accurate diagnoses and to monitor patients' medical condition, the analysis of medical records containing the psychological characteristics is crucial [15].

Radio frequency identification (RFID) technology is said to have an enormous potential to improve healthcare delivery in both hospital and home environments [14]. This technology uses radio waves to transfer data from an electronic tag attached to an object, with the purpose to identify and track the object within the infrastructure [16]. Researchers have suggested that RFID tags can be used as a mean for portable medical records [17]. The remote data link RFID feature has also been applied to the home of elderly to register movement and psychological data [17]. It has been stated that integrating RFID with a healthcare information system might improve decision-making and diagnosis because the medical staff can access accurate and real-time patient information immediately, which will help them to make quicker and more accurate decisions [17]. Patient information will be retrieved from the database using the unique ID generated by the RFID technology. This system is said to provide a cost-effective mean of increasing reliability, privacy and security in the management of the patient's healthcare records [6]. RFID is also a suitable solution when combined with other IoT-devices. Several prototypes and theories have been tested, amongst others, a prototype using RFID wristbands and tracking sensors, and wireless ECG sensor to monitor vital signs and transmit data for homestaying elderly patients with chronic diseases [17]. Another solution is to implement RFID to make smart bandages that can monitor the after-surgery patient's status. Even though several prototypes have been tested, it has been suggested that RFID applications in healthcare are still in the experimental phase [17]. In general, there are two types of RFID tags, one is active and the other one is passive. The active tag needs a battery and will transmit its ID periodically. The passive tag does not need a battery and it works by using the energy transmitted by the reader [6]. By integrating sensor technologies into passive RFID tags, a lot of completely new applications to the IoT context would be enabled, especially in e-health [11].

\subsubsection{Continuous glucose monitoring}

Continuous glucose monitoring (CGM) is an advanced way for people living with diabetes to monitor real-time glucose readings, the CGM monitors the glucose level in the user's blood and the device is connected to an insulin pump with an automated suspension of insulin infusion [18]. Several CGM systems utilizing IoT-based solutions have previously been presented. These systems collect glucose data and store it in a hospital information system, which makes it possible for medical staff to access real-time data from their patients [19]. Since the first CGM was presented and made available in 1999, several continuous glucose monitors have continued to evolve to become more accurate and user-friendly [20]. Statistics have demonstrated that CGM may reduce patient's long-term complications between $40 \%$ and $75 \%$ of the time [20]. The CGM can also be equipped with an alarm system, which may help the patient to take the correct actions when it comes to their diet, physical experience and remind them when it is time to take their medications [19]. Gia et al. [19] proposed an advanced IoT-based system for real-time glucose monitoring, body temperature and contextual data. Through this system, healthcare personnel and caregivers can easily monitor the patients at any time via a web-browser or an application on their smart-phones [19]. [21] conducted experiments using a proposed IoT-based virtual sensor to continuously measure type 2 diabetes patients. The sensor measures glucose levels in connection with additional patient information and other indicators, such as, the body mass index (BMI), blood pressure, age, presence of diabetes mellitus in first-degree relatives, and HDL cholesterol from a physical sensor connected to the IoT network. Others created glucose-monitoring applications for medical journal keeping [22].

\subsubsection{Activity- and diet tracking}

According to the World Health Organization, 30 minutes of regular, moderate-intensity activity on a daily basis, will help prevent or delay the onset of type 2 diabetes [2]. In addition, a healthy diet, avoiding sugar to achieve and maintain a healthy body weight is important [2]. Using IoT-technology such as smart watches or wristband sensors can easily monitor the patient's activity level. The smart watches can monitor walking steps taken, distance, calories burned, pace, active versus inactive time etc. Some of them also offer diet-tracking capabilities [23]. In addition, researchers developed a wearable sensor that can stick to a single tooth to track the user's diet, based on the chemical changes in their mouth. The sensors communicate with a mobile device 
through a wireless network, and transmit information on the intake of glucose, alcohol and salt [24]. The data collected from the watches/wristbands can easily be transmitted to a connected application, giving a complete overview of the patients' activity level and diet [23]. This information can also be shared with the patient's contact persons and the patient's doctor.

\subsubsection{Challenges}

Many challenging issues still need to be resolved before the concept of IoT is widely accepted. Atzoria et al. [11] claim that people will resist IoT adoptions as long as there is no public confidence that IoT will not cause serious threats and violations to their privacy [11]. In current healthcare systems, critical gaps are found in the collection of patient information and the process of transferring information to healthcare providers. The information handover among medical staff can introduce human errors, which can place the patients' health at risk. Another issue is communicating with patients that lack verbal communication skills, where the information collection problems are even more challenging [14]. Some major challenges related to security concerns, authentication and data integrity have also been discussed [11]. The IoT is extremely vulnerable to attacks for several reasons. One of the reasons is that its components are unattended most of the time [3]. This makes it easy to physically attack them. Another reason is eavesdropping. Since most of the communication is wireless, it makes eavesdropping possible [11]. Lastly, since most of the components are characterizes by low capabilities in terms of energy and computing resources, they cannot implement complex schemes that are capable of supporting high and complex security measures [11]. Data privacy, management and security are paramount considerations in the healthcare sector, with critical consequences if not managed properly $[17,12]$. IoT technology implementations have raised numerous concerns about personal data privacy and security. Even though many of today's devices use secure methods to communicate information to the cloud, they could still be vulnerable to hackers. Beyond personal data being stolen and misused, IoT devices can be used for physical harm. Several challenges still remain and must be addressed before RFID technology can be universally deployed in healthcare environments, applications and services [14]. One of the main challenges in RFID information delivery is guaranteeing security and privacy [14]. Hence, privacy and security threats could be contributing factors that slow down the adoption of RFID in healthcare [17]. Since RFID tags automatically respond to the interrogation from the reader, any person carrying an RFID tag is prone to covert physical tracking [14]. Challenges related to privacy mainly originate from counterfeiting unencrypted sensitive data within RFID tags, intercepting data during transmission, or the unauthorized access of sensitive data. Because most RFID tags rely on wireless interfaces, RFID systems may also be subject to physical attacks, such as timing attacks, fault integration and power analysis attack [17]. Efficient authentication algorithm is needed to ensure the security of smart RFID systems, the authentication focuses on the problem of well-behaved readers harvesting information from misbehaving tags (for example counterfeit ones). A problem for the basic RFID tags is that they are vulnerable to simple counterfeiting attacks [14].

Table 1. Publications per category

\begin{tabular}{|l|l|}
\hline Patient monitoring & $\begin{array}{l}{[25][26][14][15][16][17][6]} \\
{[11][27][28][29][30][21]}\end{array}$ \\
\hline $\begin{array}{l}\text { Activity- and diet } \\
\text { tracking }\end{array}$ & {$[31][23][24][25][28]$} \\
\hline $\begin{array}{l}\text { Continuous glucose } \\
\text { monitoring }\end{array}$ & $\begin{array}{l}{[18][19][20][32][29][33][29]} \\
{[21][22]}\end{array}$ \\
\hline Challenges & {$[26][11][14][12][17]$} \\
\hline
\end{tabular}

\section{Discussion}

The findings from this paper suggest that IoTtechnologies are a good solution for improving and simplifying the life of diabetes patients. As explained previously, to prevent further development of the disease, monitoring of blood glucose levels, physical activity and diet are crucial. Our findings also suggest that, different IoT-technologies can aid both patients and healthcare personnel to monitor these factors, without the need for the constant visits to the hospital and reduce the load on the usually outnumbered healthcare personnel. Networked sensors, RFID-tags, smart watches and wearable sensors are just some of the solutions that are currently available and offered. These technologies are promised to deliver a lot of benefits related to patient safety and efficiency. These solutions may help in eliminating critical errors, such as, patient misidentification, poor patient tracking, insufficient patient monitoring and poor decision-making [14]. IoTtechnologies can also assist in minimizing human errors, which are said to be one of the main reasons for medication errors [17].

The use of IoT has been instrumental in delivering more valuable real-time data to doctors, which also decreases the need for direct doctor-patient interaction [12]. This helps to improve the quality and effectiveness of service, which bring especially high value for patients with chronic conditions [7]. On the other hand, by using smartwatches to monitor activity, heart rate and diet, and using RFID technology, wearable sensors or other CGM systems to identify and track their blood glucose levels, both, patients and healthcare personnel will have access to a complete up-to-date journal of the patient's health. 
This will also enable having a complete overview that will help medical staff to decide the right treatment and timely intervention for the right patient.

Chouffani [12] states that, using IoT in the healthcare industry can be life threatening if not properly secured [12]. Hence, advanced authentication algorithms are needed to ensure the security of these systems, and it is important that both developers and managers understand the importance of this authentication process [14]. RFID technology is said to have an enormous potential to improve healthcare delivery in both hospitals and home environments [14], however, privacy and security threats are considered as barriers to the adoption of RFID technology in healthcare, and several challenges still needs to be addressed before the technology can be deployed in healthcare environments. There are, as explained in this paper, several devices and equipment that can help improve the life quality of diabetes patients. However, another challenge with using IoT in the healthcare industry is the lack of interoperability, which prevents some devices and equipment from communicating with each other [34]. Further research and development are needed in order to improve the communication between devices, and to develop worldwide protocols and communication standards for IoT in the healthcare sector. In the future, we may witness one solution for the patients, which contain all the necessary sensors and equipment into one single device, so that there is no need to have several sensors/devices attached to the body. In this case, there are also several privacy and security concerns to account for. There are, without a doubt, a lot of exciting opportunities by implementing IoT in healthcare; however, one cannot ignore the serious challenges related to the technologies. Studies have revealed that $89 \%$ of those using IoT in healthcare have suffered an IoT-related security breach [35]. These security and privacy concerns are ones of the main reasons for why people hesitate to start using IoTtechnologies in their businesses. This is especially important in the healthcare industry, due to the amount of sensitive data stored in the databases [35]. Even though there are several challenges related to privacy and security to consider, it is still believed that the technology will keep on growing and expanding. We believe that in the future, IoT will play an even more important role in the healthcare industry all over the world.

\section{Conclusions and future research}

In the future, as IoT devices are expected to become more persuasive in healthcare facilities, we will see these next-generation IoT devices bring embedded intelligent healthcare services as part of their offerings. Advancing information technologies and emerging IoT technologies have already provided numerous opportunities for the development of smarter healthcare information systems, and they will just keep on developing over the next years. This paper discusses some of the activities that can be improved and simplified by using IoT in the therapy of diabetes patients. These activities include using wearable sensors, which make it possible to gather important information about physical and mental health of the patients. In addition, RFID-tags can create a portable medical record that may provide a cost-effective mean of increasing reliability, privacy and security in the management of the patient's healthcare records. Other solutions include using continuous glucose monitoring to monitor and access real-time glucose measurements and readings, and the use of smart-watches or other wristbands for activity and diet tracking. These are only some of the available technical solutions within IoT in healthcare, and one can say with certainty that over the next decade, several new and improved devices will evolve.

Through this review, we identified that there is a research gap on privacy and security concerns related to IoT in healthcare, and that the research is more focused towards IoT applications and devices. In this industry, there is a massive amount of sensitive data stored, and this data needs to be protected and stored properly in order to achieve a successful adoption of IoT in healthcare organizations. In addition, future research should consider looking into how General Data Protection Regulation (GDPR) can affect IoT usage and practicalities in healthcare. One of the limitations of this paper is the narrow scope of IoT applications in healthcare, which means that there might exist other interesting literature that could be applied in the context of diabetes therapy but have been missed. However, we shed the light on the extant body of knowledge within our focus, which can aid researchers and practitioners to pinpoint the main research advances in the area of IoT applications for diabetes patients.

\section{References}

1. WHO: World Health Organization. (Accessed 2019) Available at: https://www.who.int/news-room/factsheets/detail/diabetes

2. Gómeza, J., Oviedob, B., Zhuma, E.: Patient Monitoring System Based on Internet of Things. Procedia Computer Science , 90-97 (2016)

3. Sajid, O., Haddara, M.: NFC Mobile Payments: Are we ready for them? In : SAI Computing Conference, London, UK, vol. Springer, pp.960-976 (2016)

4. Haddara, M., Helgesen, T.: Wireless Power Transfer Solutions for 'Things' in the Internet of Things. Advances in Intelligent Systems and Computing 880(1), 92-103 (2018)

5. Browser, J., Saxena, S., Fraser, H., Marshall, A.: A Healthy Outlook: Digital Reinvention in Healthcare., New Orchard Road Armonk, NY 10504 (2017)

6. Omar, H., Khoshnaw, A., Monnet, W.: Smart Patient Management, Monitoring and Tracking System Using Radio-Frequency Identification (RFID) Technology. IEEE EMBS Conference on Biomedical Engineering and Sciences (IECBES), 40-45 (2016) 
7. Bhatt, Y., Bhatt, C.: Internet of Things in HealthCare. Internet of Things and Big Data Technologies for Next Generation Healthcare , 13-33 (2017)

8. Webster, J., Watson, R.: Analyzing the past to prepare for the future: Writing a literature review. MIS quarterly, xiii-xxiii (2002)

9. Fernandez, F., Pallis, G.: Opportunities and challenges of the Internet of Things for healthcare. IEEE Explore, 263-266 (2014)

10. Fortino, G., Trunfio, P.: Internet of things based on smart objects: Technology, middleware and applications. SpringerLink, 1-27 (2014)

11. Atzoria, L., Lerab, A., Morabito, G.: The Internet of Things: A survey. Computer Networks, 1-19 (2010)

12. Chouffani, R.: Techtarget. (Accessed 2019 ) Available at: https://internetofthingsagenda.techtarget.com/feature Can-we-expect-the-Internet-of-Things-in-healthcare

13. Yin, Y., Zeng, Y., Chen, X., Fan, Y.: The internet of things in healthcare: An overview. Journal of Industrial Information Integration, 3-13 (2016)

14. Hu, L., Ong, D., Zhu, X., Liu, Q., Song, E.: Enabling RFID technology for healthcare: application, architecture, and challenges. Telecommunication Systems, 259-271 (2015)

15. Hassanalieragh, M., Page, A., Soyata, T., Sharma, G., Aktas, M., Mateos, G., Kantarci, B., Andreescu, S.: Health Monitoring and Management Using Internet-of-Things (IoT) Sensing with Cloud-based Processing: Opportunities and Challenges. IEEE International Conference on Services Computing, 285-292 (2015)

16. Ajami, S., Rajabzadeh, A.: Radio Frequency Identification (RFID) technology and patient safety. Journal of Research in Medical Sciences , 809-813 (2013)

17. Haddara, M., Staaby, A.: RFID Applications and Adoptions in Healthcare: A Review on Patient Safety. Procedia Computer Science , 80-88 (2018)

18. Rodbard, D.: Continuous Glucose Monitoring: A Review of Successes, Challenges, and Opportunities. Diabetes Technology \& Therapeutics, S2-3-S2-13. (2016)

19. Gia, T., Ali, M., Dhaou, I., Rahmani, A., Westerlund, T., Liljeberg, P., Tenhunen, H.: IoT-based continuous glucose monitoring system: A feasibility study. Procedia Computer Science , 327-334 (2017)

20. Zisser, H., Lane, J. E., Shivers, J. P.: Continuous Glucose Monitoring: Professional and Real Time. In : Technological Advances in the Treatment of Type 1 Diabetes. Karger (2015) 81-98

21. Mun Lee, B.: Virtual Sensor for Diabetes Meter in U-Health Service. International Journal of BioScience and Bio-Technology 6(6), 87-96 (2014)

22. Zhang, P., Schmidt, D., White, J., Mulvaney, S.: Towards Precision Behavioral Medicine with IoT: Iterative Design and Optimization of a SelfManagement Tool for Type 1 Diabetes. In : IEEE
International Conference on Healthcare Informatics (ICHI), New York, NY, USA ( 4-7 June 2018)

23. Swan, M.: Sensor Mania! The Internet of Things, Wearable Computing, Objective Metrics, and the Quantified Self 2.0. Jorunal of Sensor and Actuator Networks, 217-253 (2012)

24. Murison, M.: Health IoT: Scientists develop diet wearable - for your teeth. Internet of Business (2018)

25. Istepanian, R., Hu, S., Philip, N., Sungoor, A.: The potential of Internet of $\mathrm{m}$-health Things " $\mathrm{m}$-IoT" for non-invasive glucose level sensing. In : Annual International Conference of the IEEE Engineering in Medicine and Biology Society, Boston, USA (2011)

26. Deshkar, S., Thanseeh, R., Menon, V.: A Review on IoT based m-Health Systems for Diabetes. International Journal of Computer Science and Telecommunications 8(1), 13-18 (2017)

27. Rahman, R., Aziz, N., Kassim, M., Yusof, M.: IoTbased personal health care monitoring device for diabetic patients. In : IEEE Symposium on Computer Applications \& Industrial Electronics (ISCAIE), Langkawi, Malaysia (2017)

28. Al-Taee, M., Al-Nuaimy, W., Al-Ataby, A., Muhsin, Z., Abood, S.: Mobile health platform for diabetes management based on the Internet-of-Things. In : IEEE Jordan Conference on Applied Electrical Engineering and Computing Technologies (AEECT), Amman, Jordan (2015)

29. Jara, A., Zamora, M., Skarmeta, A.: An internet of things-based personal device for diabetes therapy management in ambient assisted living (AAL). Personal and Ubiquitous Computing 15(4), 431-440 (2011)

30. Fernández-Caramés, T., Fraga-Lamas, P.: Design of a Fog Computing, Blockchain and IoT-Based Continuous Glucose Monitoring System for Crowdsourcing mHealth. Proceedings 4(1) (2019)

31. Lee, B., Ouyang, J.: Intelligent Healthcare Service by using Collaborations between IoT Personal Health Devices. International Journal of Bio-Science and Bio-Technology 6(1), 155-164 (2016)

32. O'Connor, P., Sperl- Hillen, J., Fazio, C., Averbeck, B., Rank, B., Margolis, K.: Outpatient diabetes clinical decision support: current status and future directions. Diabetic Medicine 33(6), 734-741 (2016)

33. Fernández-Caramés, T. M., Fraga-Lamas, P.: Design of a Fog Computing, Blockchain and IoT-Based Continuous Glucose Monitoring System for Crowdsourcing mHealth. Proceedings (37) (2019)

34. Fotheringham, S.: Diabetes and the Internet of Things. Pharmaceutical Engineering (2017)

35. Srivastava, S.: Possibilities of IoT Application in the Healthcare Sector. AppInventiv.com (2019) 